\title{
A Utilização da Radiofrequência no Tratamento Fibro Edema Gelóide: Uma Revisão de Literatura
}

\author{
Fernanda da Silva Souza '; Karla Cavalcante Silva de Morais ${ }^{2}$; Nayara Alves de Sousa ${ }^{3}$; Juliana Barros Ferreira ${ }^{4}$
}

\begin{abstract}
Resumo: Objetivo: revisar a literatura sobre a eficácia da radiofrequência no tratamento do fibroedema gelóide. Metodologia: trata-se de uma revisão bibliográfica mediante a busca de artigos científicos nas plataformas Scielo e PubMed, publicados entre os anos de 2008 a 2018. Como descritores utilizou-se em inglês: "treatment" and "esthetic" or "treatmentradiofrequency" and "radiofrequencyand celulite"e o marcador boleano and.E em português: fisioterapia, celulite e radiação eletromagnética. Após aplicar filtros de pesquisa e os critérios de inclusão foram selecionados os artigos que serviram de base para a realização deste estudo. Resultados: Foram encontrados seis artigos nos meses de abril e maio, os quais foram organizados em um quadro. A literatura evidenciou eficácia da utilização da radiofrequência e melhora significativa do aspecto do fibro edema gelóide. Considerações Finais: A utilização de radiofrequência mostra-se eficaz e uma técnica promissora na terapêutica do fibro edema gelóide.
\end{abstract}

Palavras-chave: Estética. Tratamento. Celulite. Fisioterapia. Radiação Eletromagnética.

\section{The use of Radiofrequency in the Treatment of Cellulitis: A Literature Review}

\begin{abstract}
Objective: Review the literature about the efficiency of the radiofrequency on treatment the cellulitis. Methodology: it is a bibliographical review through the search of scientific articles on the platforms Scielo and PubMed, published between the years of 2008 to 2018. As descriptors has been used English: "treatment" and "esthetic" or "treatment radio frequency" and "radiofrequencyand cellulite "And the Boolean marker and.E in Portuguese: physiotherapy, cellulite and electromagnetic radiation. After applying search filters and the inclusion criteria, the articles that served as basis for the study were selected. Results: It was found six articles collected during the months April and May, which were organized in a framework. The literature evidenced the efficiency of the usage of the radiofrequency and significant improvement in the aspect of the cellulitis. Final Considerations: The usage of the radiofrequency shows efficiency and it is a promising technique in the therapy of the cellulitis.
\end{abstract}

Keywords: Esthetic. Treatment. Cellulitis. Physiotherapy. Electromagnetic Radiation.

\footnotetext{
${ }^{1}$.Graduanda em Fisioterapia pela Faculdade Independente do Nordeste-FAINOR .Vitória da Conquista/BA. Brasil. Endereço: Rua Radial Norte A, 15, Loteamento Santa Mônica, Bairro Urbis VI, Vitória da Conquista/BA. Brasil. CEP 45057320. Telefone (77)981551155 E-mail: fernandasouza38378@gmail.com

${ }^{2}$.Doutora em Desenvolvimento e Meio Ambiente/UESC. Mestre em Educação/UFBA. Docente Adjunta do Curso de Fisioterapia/UESB. Jequié/BA, Brasil. Email: nayara.sousa1@ hotmail.com

${ }^{3}$.Fisioterapeuta. Mestre em Saúde Pública pela Escola Nacional de Saúde Pública (ENSP/FIOCRUZ). Docente da Faculdade Independente do Nordeste (FANOR) e da Faculdade UNINASSAU. E-mail: karlinhakau@ hotmail.com.

${ }^{4}$.Mestre em Tecnologias em Saúde-EBMSP. Pós Graduada em Fisioterapia Dermatofuncional/UGF. Graduada em Fisioterapia pela Escola Bahiana de Medicina e Saúde Pública/EBMSP. Docente da Faculdade Independente do Nordeste- FAINOR. Endereço: Rua Sifredo Pedral Sampaio, 414, Bairro Recreio, Vitória da Conquista/BA. Brasil. CEP 45020-190. Telefone: (77)98819-2774. E mail: julianabarros@fainor.com.br
} 


\section{Introdução}

Atualmente, a imagem corporal é apresentada como o retrato no qual o próprio indivíduo se imagina, independente de formar uma imagem positiva ou com aspectos negativos (MACHADO et al., 2011, GOMES et al , 2015, GOMES, 2016). Ferreira, Lemos e Silva (2016), relatam em seu estudo que a imagem corporal tem ocupado lugar de destaque nos avanços sociais, econômicos, tecnológicos e culturais, e evidencia um maior interesse entre as mulheres, se comparado ao público masculino.

Brito e Silva (2017), afirmam que os padrões culturais e, principalmente, as mídias sociais influenciam no modo como as pessoas se preocupam com a própria autoestima, bem como com a satisfação corporal. Por conta disso, estudos têm levantado uma série de problemas relacionados à beleza corporal, e entre esses,é possível destacar o Fibro Edema Gelóide (FEG), que se associa com adiposidade localizada, flacidez muscular e aumento da massa corporal (MACHADO et al., 2011).

O FEG, popularmente chamado de celulite, é conceituado como um processo não inflamatório do tecido conjuntivo subcutâneo, caracterizado por uma infiltração edematosa que, por sua vez, provoca uma reação fibrótica (DUS, 2011).

Trata-se de um comprometimento estético que pode atingir cerca de $90 \%$ das mulheres após a puberdade, de todas as raças, tornando-se um motivo de preocupação, relacionada à estética corporal feminina, que pode trazer consequências a autoestima e comprometimento em sua vida social . (SILVA, et al., 2017).

No sexo feminino, além de fatores externos como o fumo e sedentarismo, os fatores como co-morbidades associadas e maus hábitos alimentares também contribuem para o aparecimento do FEG (SILVA, 2012).Com relação ao tratamento, têm-se as técnicas cirúrgicas, aplicações medicamentosas, terapias manuais, prática regular de atividades físicas, bons hábitos alimentares, além dos procedimentos estéticos, dentre estes, destaca-se a Radiofrequência (RF) (TAVARES et al., 2016).

A radiofrequência é descrita por Gonçalves, Rosa e Lima (2017), como uma técnica termoterapêutica que utiliza radiação no espectro eletromagnético, entre 30(Kilo- Kertz)KHz e 300(Mega-Hertz) MHz. Esse efeito térmico consegue produzir energia ondegera calor nas camadas mais profundas da pele, sem alterar a temperatura superficial, dessa forma há uma 
contração das fibras de colágeno, ativação fibroblástica, e neocolanogênse (SILVA et al., 2013).

Com o recente desenvolvimento tecnológico no âmbito da estética, a Radiofrequência, passou a ser utilizada no rejuvenescimento e flacidez agindo na derme e na hipoderme. Consiste em um procedimento não invasivo que promove melhora na nutrição e oxigenação tecidual a níveis subcutâneos, onde o calor é produzido em decorrência de uma vibração iônica promovida pela passagem da energia eletromagnética nos tecidos (BRITO, 2017).

Estudos mostram que os tratamentos com a RF é uma técnica eficaz que melhora a flacidez e a elasticidade da pele, promove a contração dos septos de tecido adiposo, e assim contribui para um melhor aspecto do FEG (TAVARES et al., 2016, FABRIZZIO et al., 2017,SARTORI, 2017,FRITZ,SALAVASTRU, GYUROVA, 2018).

Contraindica-se o uso da RF em pessoas com alteração da sensibilidade, que fazem uso de marcapasso e desfibriladores automáticos internos, além do uso do aparelho sobre todo e qualquer implante metálico, hemofílicos, gestantes. Ao se excluir essas situações a RF possui uso seguro em todos os fototipos e em todas as áreas do corpo (TRIBS, 2016).

A fisioterapia Dermatofuncional vem atuando de modo eficaz com as mais diversas técnicas para tratamento dos comprometimentos que impactam negativamente a imagem corporal do indivíduo. Além disso, tem se mostrado em evidencia no mercado, trazendo tecnologias inovadoras, com resultados satisfatórios e proporcionando a melhora na autoestima de quem se submete ao procedimento (FABRIZZIO, 2017).

Em relação aos efeitos estéticos negativos e a repercussão psicológica que o FEG pode causar, os tratamentos estéticos têm ocupado lugar de destaque por serem, na maioria das vezes de fácil aplicabilidade, e proporcionam uma melhor qualidade de vida (BAYRAKCIet al., 2010; VERAS, 2010).

Partindo desses pressupostos e pela relevância dessa temática, esse estudo propõe revisar a literatura sobre a eficácia da radiofrequência no tratamento do fibroedemagelóide. 


\section{Percurso Metodológico}

Foi realizada uma revisão bibliográfica mediante a busca de artigos científicos nas plataformas Scielo e PubMed, publicados entre os anos de 2008 a 2018, onde foram encontrados 1032 artigos.

Os descritores utilizados para a busca na plataforma eletrônica Pubmed foram: "treatment" and "esthetic" or "treatment radiofrequency and radiofrequency and celulite" e o marcador boleano "and". Para a busca no Scielo, foram utilizados os descritores em português: "fisioterapia", "celulite", "radiofrequência". Ao filtrar a pesquisa por texto completo e as publicações nos últimos 9 anos, foram selecionados 37 artigos.

Foram considerados critérios de inclusão os artigos que relatavam sobre a eficácia da RF no FEG, utilizada de forma isolada e em humanos. Foram excluídos os artigos que utilizaram outras formas de tratamento associados à RF e artigos com aplicação ou uso em animais.

Foram selecionados e utilizados seis artigos que serviram de base para esta pesquisa, e que possibilitaram a descrição dos resultados de forma qualitativa. Todos os artigos escolhidos estão disponíveis online e em texto completo.

\section{Resultados e Discussão}

Após a escolha e tradução dos artigos foi realizado um resumo com o intuito de melhor entendimento sobre o assunto e junção com a pesquisa. Em seguida foi realizada uma divisão entre os artigos separando autor/ ano, objetivo, protocolo e resultados, sendo colocados organizadamente de forma decrescente respeitando o ano de publicação (QUADRO 1). 
Quadro 1: Características dos estudos selecionados sobre Efeito da Radiofrequência no Tratamento do Fibro Edema Gelóide. Vitória da Conquista - BA. Fonte: Dados da pesquisa, 2018.

\begin{tabular}{|c|c|c|c|}
\hline $\begin{array}{c}\text { Autor/ } \\
\text { Ano }\end{array}$ & Objetivo & Protocolo & Resultados \\
\hline 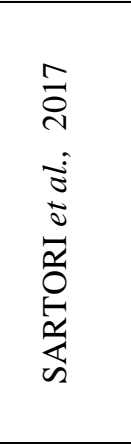 & $\begin{array}{l}\text { Analisar a eficácia do } \\
\text { equipamento de } \\
\text { radiofrequência no } \\
\text { tratamento de mulheres } \\
\text { com FEG nos graus II e } \\
\text { III na região de glúteo }\end{array}$ & $\begin{array}{l}\text { Utilizou-se o equipamento de } \\
\text { radiofrequência na região glútea, a placa de } \\
\text { acoplamento foi colocada próximo a região } \\
\text { a ser tratada e foi utilizado a manopla } \\
\text { corporal de } 35 \mathrm{~mm} \text {. A intensidade do } \\
\text { aparelho foi programada em } 100 \% \text {, foram } \\
\text { realizados movimentos circulares contínuos } \\
\text { e lentos, até que a temperatura atingisse } \\
40^{\circ} \mathrm{C} \text {. O tempo para que essa temperatura } \\
\text { fosse atingida variava entre dois e três } \\
\text { minutos. }\end{array}$ & $\begin{array}{l}\text { Melhora no grau da } \\
\text { celulite assim como } \\
\text { melhora do aspecto } \\
\text { da pele nas mulheres } \\
\text { avaliadas, } \\
\text { demonstrando efeito } \\
\text { benéfico da } \\
\text { radiofrequência para } \\
\text { o tratamento do } \\
\text { FEG. }\end{array}$ \\
\hline 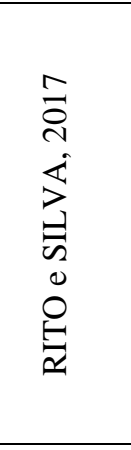 & $\begin{array}{l}\text { Averiguar os efeitos da } \\
\text { radiofrequência no } \\
\text { tratamento do } \\
\text { FibroedemaGelóide }\end{array}$ & $\begin{array}{l}\text { O procedimento foi realizado totalizando } \\
\text { em cinco sessões com intervalo de uma } \\
\text { semana entre cada uma delas. Todas as } \\
\text { sessões seguiram criteriosamente o } \\
\text { protocolo de higienização do local, } \\
\text { aplicação do gel condutor glicerinado no } \\
\text { local de tratamento, aplicação da } \\
\text { radiofrequência em toda a região glútea, } \\
\text { divididas em quadrantes, a manopla } \\
\text { utilizada foi a tripolar e o aparelho foi } \\
\text { passado até atingir a temperatura }\end{array}$ & $\begin{array}{l}\text { Comprovou-se } \\
\text { melhora na textura e } \\
\text { depressões da pele } \\
\text { associadas aos FEG. }\end{array}$ \\
\hline 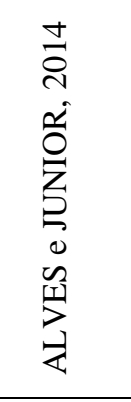 & $\begin{array}{l}\text { Avaliar os efeitos da } \\
\text { Radiofrequência (RF) no } \\
\text { tratamento do fibro } \\
\text { edema gelóide e da } \\
\text { adiposidade localizados } \\
\text { na região glútea e } \\
\text { posterior de coxa de } \\
\text { mulheres jovens e } \\
\text { sedentárias. }\end{array}$ & $\begin{array}{l}\text { As participantes foram submetidas à } \\
\text { avaliação do fibro edema gelóide, por meio } \\
\text { de fotografias, da gordura localizada, pela } \\
\text { coleta de dados antropométricos e } \\
\text { autoestima, utilizando a escala de satisfação } \\
\text { corporal. Estes instrumentos foram } \\
\text { aplicados antes e após a realização de } 3 \\
\text { sessões de RF }\end{array}$ & $\begin{array}{l}\text { As participantesdo } \\
\text { estudo obtiveram } \\
\text { melhora em todas as } \\
\text { variáveis em questão } \\
\text { (fibro edema gelóide, } \\
\text { circunferência do } \\
\text { quadril e coxas e da } \\
\text { autoestima), } \\
\text { demonstrando a } \\
\text { efetividade da RF }\end{array}$ \\
\hline 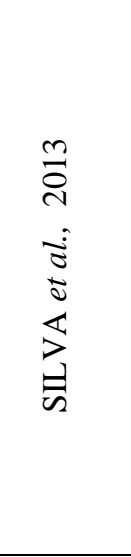 & $\begin{array}{l}\text { Avaliar os efeitos da } \\
\text { baixa temperatura da } \\
\text { radiofrequência para } \\
\text { confirmar o afinamento } \\
\text { do tecido colágeno e } \\
\text { septos interlobulares e } \\
\text { consequente melhora da } \\
\text { celulite. }\end{array}$ & $\begin{array}{l}\text { Uma amostra de oito mulheres foi usada } \\
\text { para coletar dados ultrassonográficos com } \\
\text { uma sonda de } 12 \mathrm{MHz} \text { que mediu a fibra de } \\
\text { colágeno espessura. O dispositivo foi } \\
\text { utilizado na frequência de } 0,55 \mathrm{MHz} \text { e } \\
\text { eletrodo ativo de } 3,5 \mathrm{~cm} \text { de diâmetro (área }= \\
9,61 \mathrm{~cm} 2 \text { ), foi aplicada a uma região de } 10 \\
\mathrm{~cm} 2 \text { da região glútea por } 2 \text { minutos por área } \\
\text { de eletrodo ativo, durante } 10 \text { sessões } \\
\text { quinzenais. }\end{array}$ & $\begin{array}{l}\text { O uso de } \\
\text { temperaturas mais } \\
\text { confortáveis } \\
\text { favoreceram a } \\
\text { redução da espessura } \\
\text { do septo fibroso e } \\
\text { consequente } \\
\text { melhoria da celulite, } \\
\text { evidenciada pelo } \\
\text { menor grau de } \\
\text { gravidade e } \\
\text { diminuição da } \\
\text { espessura do septo } \\
\text { interlobular. }\end{array}$ \\
\hline
\end{tabular}




\begin{tabular}{|c|c|c|c|c|}
\hline 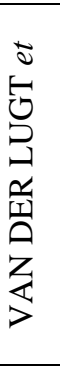 & 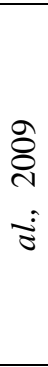 & $\begin{array}{l}\text { Avaliar a eficácia da RF } \\
\text { bipolar para o tratamento } \\
\text { da celulite. }\end{array}$ & $\begin{array}{l}\text { Foram realizadas em } 50 \text { participantes, doze } \\
\text { sessões semanais de aplicação da RF } \\
\text { bipolar, com a temperatura a } 42^{\circ} \mathrm{C} \text {, por } 12 \\
\text { minutos em cada região glútea, A melhora } \\
\text { do aspecto da celulite e a condição dos } \\
\text { tecidos foram avaliadas antes e } \\
\text { imediatamente após a primeira e décima } \\
\text { segunda sessões e } 2 \text { meses depois. }\end{array}$ & $\begin{array}{l}\text { Foi observado em } \\
\text { quase todas as } \\
\text { participantes, } \\
\text { melhora da celulite e } \\
\text { da silhueta corporal } \\
\text { na sessão final, que } \\
\text { diminuiu na } \\
\text { avaliação de } 2 \\
\text { meses. } \\
\end{array}$ \\
\hline 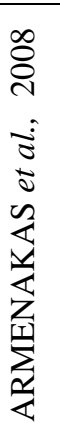 & & $\begin{array}{l}\text { Este estudo comparativo } \\
\text { cego, bilateral e cego } \\
\text { avalia a eficácia e } \\
\text { segurança de um } \\
\text { dispositivo RF unipolar } \\
\text { para melhorar a aparência } \\
\text { da celulite usando um } \\
\text { novo sistema de } \\
\text { graduação quantitativa da } \\
\text { celulite. }\end{array}$ & $\begin{array}{l}\text { Neste projeto dividido, cego e randomizado } \\
\text { estudo, } 10 \text { indivíduos ( } 32-57 \text { anos de idade) } \\
\text { com um excesso clinicamente observável de } \\
\text { gordura subcutânea e celulite grau } 2 \text { de } 4 \text { ) } \\
\text { nas coxas receberam até seis tratamentos } \\
\text { unilaterais (número de tratamentos a critério } \\
\text { do pesquisador) em intervalos de } 2 \text { semanas } \\
\text { com RF unipolar. O lado não tratado da } \\
\text { coxa serviu como controle interno. Coxas } \\
\text { tratadas foram aleatoriamente atribuído por } \\
\text { alocação alternativa. }\end{array}$ & $\begin{array}{l}\text { O dispositivo RF } \\
\text { unipolar é seguro } \\
\text { para o tratamento da } \\
\text { celulite, levando a } \\
\text { uma melhoria } \\
\text { clinicamente visível. }\end{array}$ \\
\hline
\end{tabular}

Fonte: Pesquisa dos autores.

SARTORI et al (2017), ressalta que o FEG atinge milhares de mulheres no mundo. Essa afecção é dividida em três graus: brando ou leve, moderado e grave. Esse estudo foi realizado com dez mulheres, que apresentavam fibro edema geloide em região glútea. As pacientes foram avaliadas através de uma anamnese, antropometria da região de flancos, quadril e culotes, além de fotografias para controle e averiguação da eficácia do tratamento. Após essa avaliação, as participantes foram submetidas a 10 sessões de RF, com intervalos mínimos de um dia entre uma sessão e outra. Como resultado da aplicação da técnica com a RF, percebeu-se uma melhora significativa no aspecto do FEG nas participantes.

Brito e Silva (2017), utilizaram uma amostra de mulheres que apresentavam FEG, grau II em região glútea. Realizou-se uma anamnese, registro fotográfico e antropométrico das participantes e foi aplicado um protocolo de cinco sessões de RF, com intervalo de uma semana entre uma sessão e outra. As sessões foram realizadas com um aparelho de RF comum a manopla tripolar, com movimentos circulares, até atingir a temperatura de 38 graus centígrados $\left({ }^{\circ} \mathrm{C}\right)$ por 2 dois minutos. Após o final das cinco sessões, e verificação dos dados de comparação iniciais e finais, constatou-se que os efeitos da RF no tratamento do FEG são positivos.

Alves e Junior (2014) relatam sobre a efetividade da RF no tratamento do FEG e da adiposidade, localizados na região glútea e posterior da coxa. Mostram que a fisioterapia dermatofuncional vem se estabelecendo, pois propõe a melhora físico funcional das alterações 
endócrino-metabólicas e dermatológicas. Este estudo foi realizado em 3 mulheres, onde as participantes foram avaliadas, fotografadas, tiveram seus dados antropométricos coletados e responderam a uma escala de satisfação corporal. Esses instrumentos foram aplicados antes e após a realização de 3 sessões de RF. As voluntárias relataram e comprovaram através dos dados coletados que ocorreu uma melhora significativa na redução de medidas, com melhora do FEG, redução das medidas do quadril e das coxas e relataram satisfação com os resultados.

SILVA et al (2013),ressaltam que a RF é um recurso voltado para aplicações na derme e que possui uma gama de efeitos fisiológicos, porém, a falta de padronização nos tratamentos gera controvérsias sobre seu uso. Os autores desenvolveram um estudo onde foram avaliados os efeitos da baixa temperatura na RF, utilizando faixas de temperaturas menores que $36^{\circ} \mathrm{C}$, para confirmar a diminuição do tecido de colágeno e dos septos interlobulares e consequente melhora da celulite. A amostra foi constituída por oito mulheres, como coleta de imagem foi utilizado um aparelho de ultrassom comum a sonda de $12 \mathrm{MHz}$, que mensurou a espessura da fibra de colágeno. A aplicação após atingir a temperatura preconizada, foi na região glútea por 2 minutos, por área de eletrodo ativo, durante 10 sessões quinzenais. Concluiu-se que o uso de temperaturas mais confortáveis favoreceu a redução da espessura do septo fibroso e consequente melhora da celulite.

O estudo de VAN DER LUGT et al (2009), foi realizado em cinquenta mulheres com idade entre 24 e 58 anos, fototipos II e V. As participantes apresentavam celulite localizadas em várias áreas do corpo, especialmente nos membros inferiores e em região glútea. $\mathrm{O}$ grau do FEG foi estabelecido seguindo a caracterização de Rossi, que foi classificadocomo grau 3. Para avaliação foi utilizada uma escala de satisfação, fotografias, avaliação da área da celulite por um software de profilometria de computador tridimensional (3-D), que mensura a profundidade das depressões e rugosidade na pele, além da avaliação histológica da região (15 das 50 participantes concordaram em se submeter a biópsias de tecido) que foi realizada antes e depois da primeira sessão, após a décima segundae dois meses após a aplicação da RF. Foi utilizada a RF bipolar, com temperatura de $42^{\circ} \mathrm{C}$, durante 12 minutos em cada região glútea demarcada, com uma frequência de tratamento de uma vez na semana, durante 12 sessões semanais. Os resultados mostraram que a maioria das participantes ficaram satisfeitase notaram uma melhora nas características da pele, com menos depressões superficiais, e os achados histológicos mostraram quebra dos adipócitos. 
Armenakas et al (2008), avaliaram a eficácia e segurança de um dispositivo de RF unipolar com temperatura entre $40^{\circ} \mathrm{C}$ a $43^{\circ} \mathrm{C}$, para melhorar a aparência da celulite.Esse estudo experimental,cego e randomizado,foirealizado em 10 participantes com fototipo I,com idade entre 32 a 57 anos, que apresentavam excesso de gordura subcutânea e celulite grau 2 em região das coxas. O protocolo de tratamento foi realizado de forma unilateral em uma das coxas, e a coxa contralateral serviu como controle. As áreas a serem tratadas foram marcadas e fotografadas. As participantes receberam até seis tratamentos unilaterais com intervalo de duas semanas. Os resultados foram avaliados por meio da escala likert de 4 pontos, por meio dos registros fotográficos e escala de classificação de celulite. O acompanhamento deste estudo deu-se em um e três meses após o tratamento final. Os resultados apresentados mostraram queo dispositivo RF unipolar é seguro para o tratamento da celulite e leva a uma melhora clinicamente visível do FEG.

\section{Considerações Finais}

A utilização de Radiofrequência (RF) mostra-se eficaz e com resultadospromissoresno aspecto do Fibro Edema Gelóide (FEG). Diante disso, os tratamentos estéticos têm ocupado lugar de destaque por serem, na maioria das vezes de fácil aplicabilidade, e proporcionarem uma melhor qualidade de vida e autoestima aos portadores dessa afecção.

Diante desta revisão, acredita-se que ainda existe uma falta de padronização no método de utilização dos aparelhos de radiofrequência. No entanto, estudos clínicos randomizados e controlados com acompanhamento de followup devem ser realizados.

\section{Referências}

ABE, H.T. e FERREIRA, L. L. Tratamento do Fibro Edema Gelóide com Radiofrequência.Revista Pesquisa em Fisioterapia. 2014 Dez;4(3):206-214

ALVES, A.C.R. e JUNIOR, A.J.C. A efetividade da radiofrequência no tratamento do fibro edema gelóide e da adiposidade localizada na região glútea e região posterior da coxa. Estudos, Goiânia,v. 41, n. 2, p. 359-468, 1br./jun. 2014 
ARMENAKAS, M.A. et al., Unipolar radiofrequency treatment to improve the appearance of celulite.JournalofCosmeticand Laser Therapy. 2008

BAYRAKCI T. V, AKBAYRAK T, BAKAR Y, KAYIHAN H, ERGUN N.Effectsofmechanicalmassage, manuallymphaticdrainageandconnectivetissuemanipulationtec hniquesonfatmass in womenwithcellulite.J EurAcadDermatolVenereol; 2010 Feb; 24(2):138-42. PubMed ID: 19627407

BRITO, J. Q. A.; SILVA, A. P. O. Estudo de Caso Sobre os Efeitos da Radiofrequência no Tratamento do Fibro Edema Gelóide. Id onLine Rev. Psic. V.11, N. 35. Maio/2017 - ISSN 1981-1179

DUS, L. M. S. dos. A utilização da endermoterapia associado à cosmetologia no tratamento da flacidez facial - uma proposta terapêutica. Dissertação (Bacharelado), Universidade do Extremo Sul Catarinense, UNESC. Criciúma, junho de 2011

FABRIZZIO, et al. Análise comportamental do tecido adiposo frente ao tratamento de radiofrequência: revisão bibliográfica. Revista Saúde em Foco - Edição no 9 - Ano: 2017

FERREIRA, J. B.; LEMOS, L. M. A.; SILVA, T. R. da. Qualidade de vida, imagem corporal e satisfação nos tratamentos estéticos. RevistaPesquisaemFisioterapia. 2016 Nov;6(4):402-410

FRITZ, K., SALAVASTRU, C., GYUROVA, M. Clinical evaluation of simultaneously applied monopolar radiofrequency and targeted pressure energy as a new method for noninvasive treatment of cellulite in postpubertal women. J CosmetDermatol.2018;17:361-364

GOMES, T. B. Set al. IMAGEM CORPORAL E IMAGEM GENITAL FEMININA. Catussaba-Revista Científica da Escola da Saúde.v. 2, n. 4, p.37-42, fev/mai. 2015 .

GOMES, T. B.S. Associação entre imagem corporal e imagem genital de mulheres matriculadas em academias: um estudo observacional. 2016. 55 f. Dissertação (Mestrado) Curso de Tecnologias em Saúde, Escola Bahiana de Medicina e Saúde Pública, Salvador, 2016.

GONÇALVES, M. F.; ROSA, A. N. da.; LIMA, F. V. Tratamento do fibro edema gelóide no glúteo com a utilização da radiofrequencia e endermoterapia. Monografia, Universidade do Sul de Santa Catarina - UNISUL, Santa Catarina - RS, 2017

LUGT etal.A multicenter study of cellulite treatment with a variable emission radio frequency system.DermatologicTherapy, Vol. 22, 2009, 74-84

MACHADO, G. C. et al. Análise dos efeitos do ultrassom terapêutico e da eletrolipoforese nas alterações decorrentes do fibroedemageloide. Fisioter. Mov., Curitiba, v. 24, n. 3, p. 471-479, jul./set. 2011

MASCARO, S. F. de. Tipologias de projetos de pesquisa de métodos mistos: análise classificatória das teses de doutorado dos programas de pós-graduação em geociências no 
brasil. Dissertação (Doutorado), Instituto de Geociências e Ciências exatas Campus de Rio Claro, Universidade Estadual Paulista, Rio Claro, 2015

PETROSKI, E.L. et al. Motivos e Prevalência da Insatisfação com a Imagem Corporal e Adolescentes. TEMAS LIVRES FREE THEMES. 2013.

SARTORI, D.V.B. Verificação da eficácia da radiofrequência em mulheres com fibro edema gelóide em região de glúteo.REVISTA INSPIRAR. Edição 41 - Volume 12 - Número 1 JAN/FEV/MAR - 2017

SECCHI, K. Percepção da Imagem Corporal e Representações Sociais do Corpo. Psicologia: Teoria e PesquisaAbr-Jun 2009, Vol. 25 n. 2, pp. 229-236.

SILVA, P. C. O. de. Os efeitos da endermologia no tratamento do fibro edema gelóide: artigo de atualização. Dissertação (Especialização), Pontifícia Universidade Católica de Goiás, Goiás, 2012

SILVA, R. M. V. et al. Avaliação do grau do fibro edema gelóide utilizando um sensor de infravermelho. Revista da Saúde e Biotecnologia. 2017 jul-out;1(1):18-30.

SILVA, R.M.V.Effect of Capacitive Radiofrequency on the Fibrosis of Patients with Cellulite.Hindawi Publishing Corporation.Dermatology Research and Practice Volume 2013, Article ID 715829, 6 pages.

TAVARES, I. S. et al. RECURSOS FISIOTERAPÊUTICOS UTILIZADOS NO TRATAMENTO DO FIBRO EDEMA GELÓIDE (FEG). Revista Científica da Faculdade de Educação e Meio Ambiente. 7(2): 45-58, jul.-dez., 2016

TRIBS, T. L. T.; DELFINO, M. M. Tratamento para fibro edema gelóide com uso de radiofrequência e massagem modeladora. VII Congresso de IniciaçãoCientíficaFEPI.Itajubá/MG, Out. 2016

VERAS, A. L. L. Development and construction of body image in contemporary: a cognitivebehavioral perspective. Revista brasileira de terapias cognitivas, 2010 volume $6 n^{\circ}$

\section{Como citar este artigo (Formato ABNT):}

SOUZA, Fernanda da Silva; MORAIS, Karla Cavalcante S. de; SOUSA, Nayara Alves de; FERREIRA, Juliana Barros. A Utilização da Radiofrequência no Tratamento Fibro Edema Gelóide: Uma Revisão de Literatura. Id on Line Revista Multidisciplinar e de Psicologia, 2018, vol.12, n.41, p.77-86. ISSN: 1981-1179.

Recebido: 29.05 .2018

Aceito: 09.06.2018 